\title{
@Emerald|Insight
}

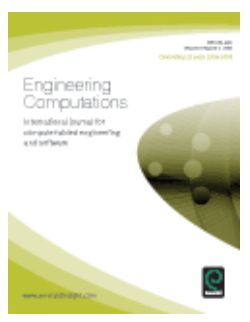

\section{Engineering Computations}

ISSN: 0264-4401

Online from: 1984

Current Issue Available Issues Most Cited Most Read ToC Alert RSS

Add to favorites
Morphological, Structural and Functional

Modeling and CAD-Centric Information

Exchange for Complex Collaborative Design Efforts in Industry

Editor(s): Bart H.M. Gerritsen, Professor Imre Horvath

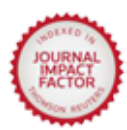

This title is indexed in Scopus
Next Article

NextArticle

\section{Cite! Sิ Save to}

\section{Sensitivity analysis in optimized parametric curve fitting}

\section{Article Options and Tools}

View: HTML PDF

References (24)

Add to Marked List Download Citation Track Citations

Q Reprints \& Permissions

Oscar E Ruiz (Laboratorio de CAD CAM CAE, Universidad EAFIT, Medellín, Colombia)

Camilo Cortes (Laboratorio de CAD CAM CAE, Universidad EAFIT, Medellin, Colombia)

Diego A Acosta (Grupo de Investigación DDP, Universidad EAFIT, Medellín, Colombia)

Mauricio Aristizabal (Laboratorio de CAD CAM CAE, Universidad EAFIT, Medellín, Colombia)

Citation:

Oscar E Ruiz, Camilo Cortes, Diego A Acosta , Mauricio Aristizabal , (2015) "Sensitivity analysis in optimized parametric curve fitting", Engineering Computations, Vol. 32 Iss: 1, pp.37 - 61

DOI

http://dx.doi.org/10.1108/EC-03-2013-0086

Downloads:

The fulltext of this document has been downloaded 172 times since 2015

Abstract:

\section{Purpose}

- Curve fitting from unordered noisy point samples is needed for surface reconstruction in many applications In the literature, several approaches have been proposed to solve this problem. However, previous works lack formal characterization of the curve fitting problem and assessment on the effect of several parameters (i.e. scalars that remain constant in the optimization problem), such as control points number $(m)$, curve degree $(b)$, knot vector composition $(U)$, norm degree $(k)$, and point sample size $(r)$ on the optimized curve reconstruction measured by a penalty function $(f)$. The paper aims to discuss these issues.

\section{Design/methodology/approach}

- A numerical sensitivity analysis of the effect of $m, b, k$ and $r$ on $f$ and a characterization of the fitting procedure from the mathematical viewpoint are performed. Also, the spectral (frequency) analysis of the derivative of the angle of the fitted curve with respect to $u$ as a means to detect spurious curls and peaks is explored.

\section{Findings}

- It is more effective to find optimum values for $m$ than $k$ or $b$ in order to obtain good results because the topological faithfulness of the resulting curve strongly depends on $m$. Furthermore, when an exaggerate number of control points is used the resulting curve presents spurious curls and peaks. The authors were able to detect the presence of such spurious features with spectral analysis. Also, the authors found that the method for curve fitting is robust to significant decimation of the point sample.

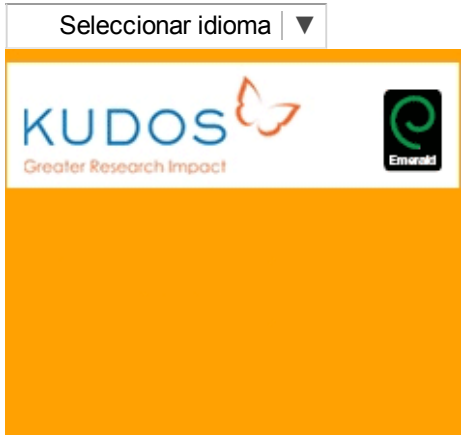

\begin{tabular}{|l|l|l|}
\hline Most Read Most Cited Related \\
\hline
\end{tabular}

The most popular articles from this title:

Last Year Last 7 Days

A NOVEL AUTOMATIC IDENTIFICATION SYSTEM FOR THREE-DIMENSIONAL MULTI-BLOCK SYSTEMS

An integrated software environment for multi-disciplinary computational engineering

Topological analysis of eigenvalues in engineering computations

Accuracy and stability for integration of Jaumann stress rate equations in spinning bodies

SIMULATION OF INDUSTRIAL FORGING OF AXISYMMETRICAL PARTS See More

\section{KUDOS}

\section{SENSITIVITY CURVE FITTING POINT CLOUDS}

What's it about?

Given a set of points sampled resembling a planar curve, this algorithm allows to find a smooth mathematical representation of the resembled curve. 
- The authors have addressed important voids of previous works in this field. The authors determined, among the curve fitting parameters $m, b$ and $k$, which of them influenced the most the results and how. Also, the authors performed a characterization of the curve fitting problem from the optimization perspective. And finally, the authors devised a method to detect spurious features in the fitting curve.

Practical implications

- This paper provides a methodology to select the important tuning parameters in a formal manner.

Originality/value

- Up to the best of the knowledge, no previous work has been conducted in the formal mathematical evaluation of the sensitivity of the goodness of the curve fit with respect to different possible tuning parameters (curve degree, number of control points, norm degree, etc.).

Keywords:

Sensitivity analysis, Minimization, Noisy point sample, Parametric curve fitting, Reverse engineering

Publisher:

Emerald Group Publishing Limited

Why is it important?

In many reverse engineering applications, the primary data is a point sample. However, a mathematical smooth representation is needed to express a shape and to execute downstream applications.

This information has been added on Kudos by the following: Professor OSCAR E RUIZ

Read more on Kudos..

\section{Further Information}

About the Journal

Sample Articles

Purchase Information

Editorial Team

Write for this journal

Share

\section{About Emerald}

- About us

- Company information

- Working for Emerald

- Contact us

- How to find us

() Emerald Group Publishing Limited

\section{(i) Policies \& Information}

- Cookie Policy

- Privacy Policy

- Copyright Policy

- Industry Standards

- Digital Preservation

- Accessibility

\section{Emerald Websites}

- Emerald Group Publishing

- Emerald Management First

- Emerald Bookstore

- Emerald Careers

- The Emerald Foundation 